\title{
CURVILINEAR SQUEEZE FILM BEARING WITH POROUS WALL LUBRICATED BY A RABINOWITSCH FLUID
}

\author{
A. WALICKA*, E. WALICKI, P. JURCZAK and J. FALICKI \\ University of Zielona Góra, Faculty of Mechanical Engineering \\ ul. Szafrana 2, 65-516 Zielona Góra, POLAND \\ E-mails: A.Walicka@ijame.uz.zgora.pl; E.Walicki@ijame.uz.zgora.pl \\ P.Jurczak@ibem.uz.zgora.pl; J.Falicki@ibem.uz.zgora.pl
}

\begin{abstract}
The present theoretical analysis is to investigate the effect of non-Newtonian lubricant modelled by a Rabinowitsch fluid on the performance of a curvilinear squeeze film bearing with one porous wall. The equations of motion of a Rabinowitsch fluid are used to derive the Reynolds equation. After general considerations on the flow in a bearing clearance and in a porous layer using the Morgan-Cameron approximation the modified Reynolds equation is obtained. The analytical solution of this equation for the case of a squeeze film bearing is presented. As a result one obtains the formulae expressing pressure distribution and load-carrying capacity. Thrust radial bearing and spherical bearing with a squeeze film are considered as numerical examples.
\end{abstract}

Key words: non-Newtonian fluid, Rabinowitsch model, curvilinear squeeze film bearing, porous layer.

\section{Introduction}

Squeeze film phenomena play an important role in many areas of engineering and applied sciences such as: polymer processing, metal processing, pressure die casting, lubrication technology, bioengineering, etc. In lubrication technology, the squeeze film behaviour results from too lubricated, generally curvilinear surfaces of revolution, approaching each other with a velocity normal to these surfaces on their common symmetry line. Classical studies of squeeze film phenomena between approaching surfaces are presented by Wu using a Newtonian fluid [1].

In recent years, tribologists have done a great deal of work of non-Newtonian lubricants in squeeze film bearings. The viscosity of these kinds of lubricants displays a non-linear relationship between the shear stress and shear strain rate. There are many known formulae to model this relationship. One of the first was power-series development and in consequence the polynomials were suggested. The polynomial given by Kraemer and Williamson [2] which was later independently proposed by Wissenberg's student Rabinowitsch [3] should be cited here. In the sixties of the past century Rotem and Shinnar [4] returned to the polynomial representation proposing their own model similar to the one of Rabinowitsch.

Theoretical considerations and some ranges of experiments carried out by Wada and Hayashi $[5,6]$ indicated on good usefulness the Rabinowitsch fluid to modelling various lubrication problems. These problems have been analyzed by many investigators, for instance as journal bearings were studied by Wada and Hayashi [5, 6], Swamy et al. [7], Rajalingham et al. [8], Sharma et al. [9], hydrostatic thrust bearing by Singh et al. [10], squeeze film bearings by Hashimoto and Wada [11], Lin [12], Lin et al. [13]. More general lubrication problems include hybrid bearings modelled by two generally non-coaxial surfaces of revolution which can work simultaneously as journal and/or thrust bearings. Some theoretical considerations about these bearings may be found in the works given by Walicka et al. [14, 15], Ratajczak et al. [16], Walicka and Walicki [17]; these authors considered both externally pressurized bearings with and without rotational inertia

\footnotetext{
${ }^{*}$ To whom correspondence should be addressed
} 
and squeeze film bearings lubricated by the Rotem-Shinnar fluid. From the results of all the papers referred to above, it follows that the non-Newtonian lubricants properties affect the bearing performance significantly.

The extensive use of porous bearings is found in industry for a long time because of their low cost and reduced lubricant requirement. Basing on Darcy model of flow through porous medium Morgan and Cameron [18] first presented theoretical research on these bearings.

The behaviour of various types of porous bearings, such as squeeze films ( $\mathrm{Wu},[1])$, externally pressurized bearings (Gupta et al. [19]), journal bearings (Prakash and Vij, [20]) and slider bearings (Uma, [21]) were considered. Of late, the use of non-Newtonian fluids as lubricants in porous bearings has gained importance in modern industry. From many studies the works given by: Walicka [22] which contains considerations on the inertia effects in rough porous squeeze film bearing with power-law lubricant, Walicka [23] on the porous squeeze film bearings with viscoplastic Shulman's type lubricant, Walicka and Jurczak [24] who considered pressure distribution in squeeze film bearing lubricated by Vočadlo viscoplastic fluid can be mentioned.

In this paper the Rabinowitsch fluid model is used to describe the non-Newtonian behaviour of a lubricant in a squeeze film bearing between two curvilinear surfaces of revolution (with one porous wall). The modified Reynolds equation is derived and its general solution for the curvilinear thrust bearing is presented. The analysis is based on the assumption that the porous matrix of the porous wall consists of a system of capillaries of very small radii which allows a generalization of the Darcy law and use of the Morgan-Cameron approximation for the flow in a porous layer.

\section{Derivation of the Reynolds equation for a Rabinowitsch fluid}

It may be assumed that lubricating oils, with a viscosity index improver added, exhibit the same characteristics as non-Newtonian fluids. Rotem and Shinnar [4] proposed a method for expressing empirically the relation between the stress and the shear rate as

$$
\frac{d \gamma}{d t}=\frac{\tau}{\mu}\left(1+\sum_{i=1}^{n} k_{i} \tau^{2 i}\right)
$$

Retaining only the first order term $(i=1)$ the above equation reduces to Rabinowitsch model [3]

$$
\mu \frac{d \gamma}{d t}=\tau+k \tau^{3}
$$

Typical flow curves are shown in Fig.1. Since $\mu$ is the tangent at the original point of the flow curves, shown in Fig. 1, $\mu$ is the initial viscosity. If the values of $\mu$ do not vary, the non-linearity of the flow curve increases with the value of $k$, which means the coefficient of plasticity. In non-Newtonian fluids $k \neq 0$ and in Newtonian fluids $k=0$. Therefore, in Newtonian fluids, the initial viscosity becomes the viscosity given by Newton's law.

The three-dimensional notation of Eq.(2.2) may be expressed as [25]

$$
\mu \boldsymbol{A}_{1}=\Lambda\left(1+k \Lambda^{2}\right)
$$

where

$$
\Lambda=\left[\frac{1}{2} \operatorname{tr}\left(\mathbf{\Lambda}^{2}\right)\right]^{\frac{1}{2}}
$$


is the magnitude of the second-order shear stress tensor $\boldsymbol{\Lambda}$, but $\boldsymbol{A}_{l}$ is the first Rivlin-Ericksen kinematic tensor.

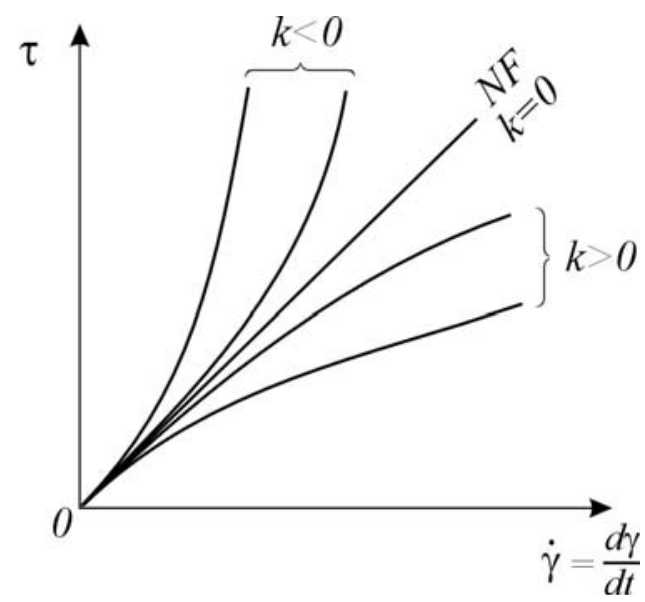

Fig.1. Flow curves of a Rotem-Shinnar fluid of the first order $(i=1)$; the symbol NF means a Newtonian fluid.

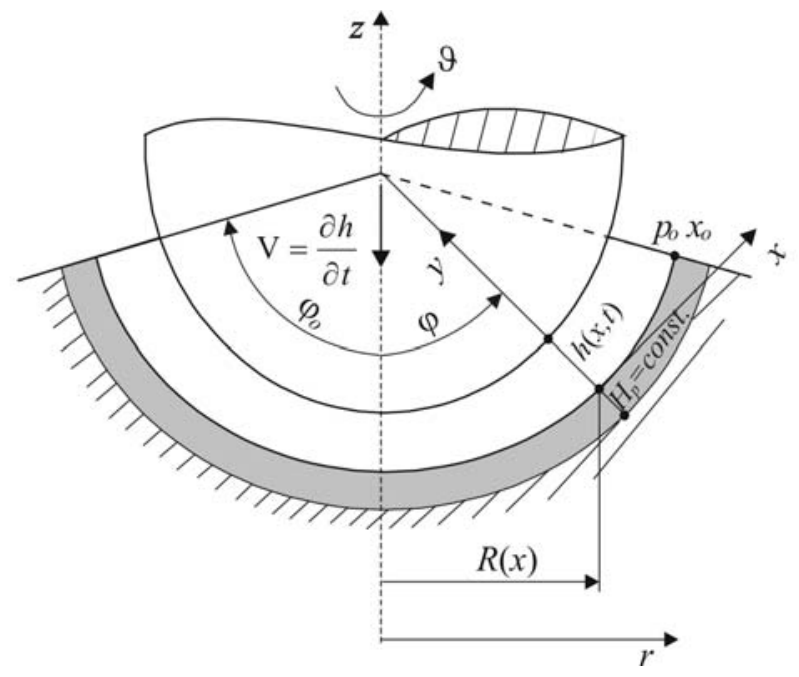

Fig.2. Geometry of a curvilinear squeeze film bearing.

Let us consider a squeeze film bearing with a curvilinear profile of the working surfaces shown in Fig. 2 in a cylindrical system of coordinates $r, \vartheta, z$. To facilitate the further considerations let us introduce an intrinsic curvilinear orthogonal coordinate system $x, \vartheta, y$ linked with the upper surface of a porous layer which is also presented in Fig.2. The upper bound of a porous layer is described by the function $R(x)$ which denotes the radius of this bound. The bearing clearance thickness is given by the function $h(x, t)$, while the porous layer thickness is given by $H_{p}=$ const. Taking into account the considerations of the works (Walicka, [25]; Walicki, [26]) one may present the equation of continuity and the equations of motion of a Rabinowitsch fluid for axial symmetry in the form

$$
\frac{1}{R} \frac{\partial\left(R v_{x}\right)}{\partial x}+\frac{\partial v_{y}}{\partial y}=0
$$




$$
\frac{\partial \Lambda_{x y}}{\partial y}=\frac{\partial p}{\partial x}, \quad \frac{\partial p}{\partial y}=0
$$

Constitutive equation (2.3) ${ }_{1}$ takes the form

$$
\mu \frac{\partial v_{x}}{\partial y}=\Lambda_{x y}+k \Lambda_{x y}^{3}
$$

The problem statement is complete after specification of boundary conditions. These conditions for velocity component are stated as follows

$$
\begin{aligned}
& v_{x}(x, 0, t)=0, \quad v_{x}(x, h, t)=0, \\
& v_{y}(x, 0, t)=V_{H}, \quad v_{y}(x, h, t)=\frac{\partial h}{\partial t}=\dot{h}
\end{aligned}
$$

where $V_{H}$ is the velocity of a throughflow on the upper bound of the porous layer. Here and in that follows it is assumed - as the first approximation - that this velocity is directed along the normal (along the $y$ coordinate) to the upper surface of the porous layer.

Solving the equations of motion (2.4), (2.5) and taking into account the constitutive equation (2.6) one obtains the Reynolds equation (detailed solution may be found in works $[25,26]$ )

$$
\frac{1}{R} \frac{\partial}{\partial x} R h^{3}\left[\frac{\partial p}{\partial x}+\frac{3}{20} k h^{2}\left(\frac{\partial p}{\partial x}\right)^{3}\right]=12 \mu\left(\frac{\partial h}{\partial t}-V_{H}\right)
$$

for a lubricating fluid of the Rabinowitsch type. If $k=0$, the above equation is identical to the Reynolds equation for Newtonian lubricant [26].

\section{Modified Reynolds equation for a bearing with a porous pad}

To solve Eq.(2.9) let us study the flow of a type Rabinowitsch fluid in the porous layer. Assume that this layer consists a system of capillaries with an average radius $r_{c}$ and porosity $\varphi_{p}$. Let the porous layer be homogeneous and isotropic and let the flow within the layer satisfy the modified Darcy's law. Thus one has $[25,27]$

$$
\begin{aligned}
& \overline{\mathrm{v}}_{x}=\frac{\Phi_{p}}{\mu}\left(-\frac{\partial \bar{p}}{\partial x}\right)+\frac{\Phi_{p}}{\mu} \frac{k r_{c}^{2}}{\sigma}\left(-\frac{\partial \bar{p}}{\partial x}\right)^{3}, \\
& \overline{\mathrm{v}}_{y}=\frac{\Phi_{p}}{\mu}\left(-\frac{\partial \bar{p}}{\partial y}\right)+\frac{\Phi_{p}}{\mu} \frac{k r_{c}^{2}}{\sigma}\left(-\frac{\partial \bar{p}}{\partial y}\right)^{3}
\end{aligned}
$$

where $\overline{\mathrm{v}}_{x}, \overline{\mathrm{v}}_{y}$ are velocity components in the porous layer and 


$$
\Phi_{p}=\frac{\varphi_{p} r_{c}^{2}}{8}
$$

is the permeability of the porous layer but $\varphi_{p}$ is the coefficient of porosity.

Since the cross velocity component $\bar{v}_{y}$ must be continuous at the porous wall-fluid film interface and must be equal to $V_{H}$, we have then - by virtue of Eqs (2.9) and (3.1) - the following form of the modified Reynolds equation

$$
\frac{1}{R} \frac{\partial}{\partial x} R h^{3}\left[\frac{\partial p}{\partial x}+\frac{3}{20} k h^{2}\left(\frac{\partial p}{\partial x}\right)^{3}\right]=12 \mu\left[\frac{\partial h}{\partial t}-\left.\frac{\Phi_{p}}{\mu}\left\{\left(-\frac{\partial \bar{p}}{\partial y}\right)+\frac{k r_{c}^{2}}{6}\left(-\frac{\partial \bar{p}}{\partial y}\right)^{3}\right\}\right|_{y=0}\right]
$$

Using the Morgan-Cameron approximation $[18,20]$ one obtains (see Appendix A)

$$
\left.\left\{\left(-\frac{\partial \bar{p}}{\partial y}\right)+\frac{k r_{c}^{2}}{6}\left(-\frac{\partial \bar{p}}{\partial y}\right)^{3}\right\}\right|_{y=0}=-\frac{H_{p}}{R} \frac{\partial}{\partial x} R\left\{\left(-\frac{\partial p}{\partial x}\right)+\frac{k r_{c}^{2}}{6}\left(-\frac{\partial p}{\partial x}\right)^{3}\right\}
$$

When formula (3.4) is inserted into Eq.(3.3) the modified Reynolds equation takes the form

$$
\frac{1}{R} \frac{\partial}{\partial x} R\left[\left(h^{3}+\frac{3}{2} \varphi_{p} r_{c}^{2} H_{p}\right) \frac{\partial p}{\partial x}+\frac{3 k}{20}\left(h^{5}+\frac{5}{3} \varphi_{p} r_{c}^{4} H_{p}\right)\left(\frac{\partial p}{\partial x}\right)^{3}\right]=12 \mu \frac{\partial h}{\partial t}
$$

for the squeezed flow in a clearance between two surfaces revolution.

\section{Solutions to the modified Reynolds equation}

To find the solution to the Eq.(3.5) consider the case of the Rabinowitsch fluid of frequent occurrence for which the factor $k \Lambda_{x y}^{2}<<1$; the value of this factor indicates that the solutions to the Reynolds equation (3.5) may be searched in a form of the sum $[14,15,28]$

$$
p=p^{(0)}+p^{(1)} .
$$

Assuming that $p^{(l)}<<p^{(0)}$ and substituting Eq.(4.6) into Eq.(4.5) we arrive at two linearized equations, the first one

$$
\frac{1}{R} \frac{\partial}{\partial x}\left\{R\left[h^{3}+\frac{3}{2} \varphi_{p} r_{c}^{2} H_{p}\right] \frac{\partial p^{(0)}}{\partial x}\right\}=12 \mu \frac{\partial h}{\partial t}
$$

and the other 


$$
\begin{aligned}
& \frac{1}{R} \frac{\partial}{\partial x}\left\{R\left[h^{3}+\frac{3}{2} \varphi_{p} r_{c}^{2} H_{p}\right] \frac{\partial p^{(1)}}{\partial x}\right\}= \\
& =-\frac{3 k}{20} \frac{1}{R} \frac{\partial}{\partial x}\left\{R\left[h^{5}+\frac{5}{3} \varphi_{p} r_{c}^{4} H_{p}\right]\left[\frac{\partial p^{(0)}}{\partial x}\right]^{3}\right\} .
\end{aligned}
$$

The boundary conditions for pressure are

$$
\left.\frac{\partial p^{(0)}}{\partial x}\right|_{x=0}=0, \quad p^{(0)}\left(x_{o}\right)=p_{o},\left.\quad \frac{\partial p^{(I)}}{\partial x}\right|_{x=0}=p^{(l)}\left(x_{o}\right)=0 .
$$

The solutions of Eqs (4.2) and (4.3) are given, respectively, as follows

$$
p(x, t)=p_{o}-12 \mu\left[F_{o}-F(x, t)\right]
$$

where

$$
\begin{aligned}
& F(x, t)=I(x, t)-\frac{108 k \mu^{2}}{5} J(x, t), \quad F_{o}=F\left(x_{o}, t\right), \\
& I(x, t)=\int \frac{\int R \frac{\partial h}{\partial t} d x}{R\left[h^{3}+\frac{3}{2} \varphi_{p} r_{c}^{2} H_{p}\right]} d x, \\
& J(x, t)=\int\left\{\frac{\left[h^{5}+\frac{5}{3} \varphi_{p} r_{c}^{4} H_{p}\right]}{R^{3}\left[h^{3}+\frac{3}{2} \varphi_{p} r_{c}^{2} H_{p}\right]^{4}}\left[\int R \frac{\partial h}{\partial t} d x\right]^{3}\right\} d x .
\end{aligned}
$$

The load-carrying capacity is defined by

$$
N=2 \pi \int_{0}^{x_{o}}\left(p-p_{o}\right) R \cos \varphi d x
$$

the sense of angle $\varphi$ arises from Fig.2.

\section{Radial thrust bearing with squeezed film}

Let us consider a radial thrust bearing with squeezed film of a Rabinowitsch lubricant modelled by two parallel disks (Fig.3). 


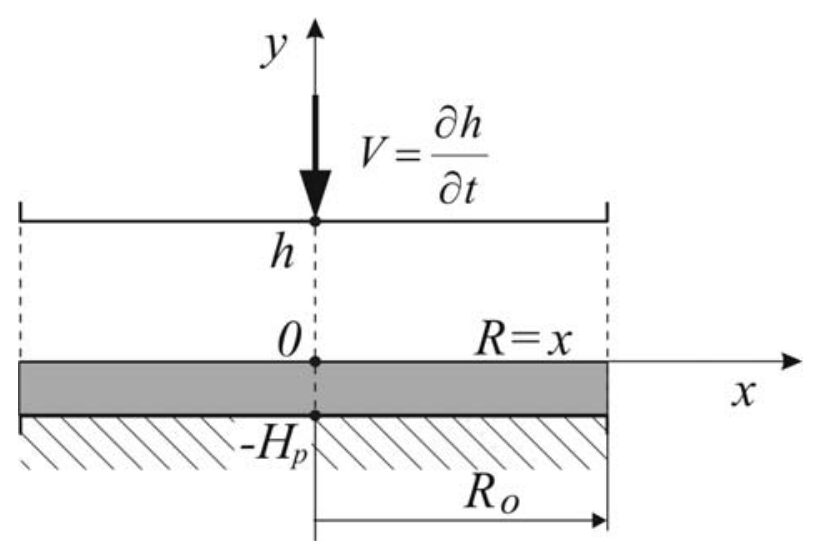

Fig.3. Squeeze film in a radial thrust bearing.

Introducing the following parameters

$$
\begin{aligned}
& \tilde{x}=\frac{x}{R_{o}}, \quad x=R, \quad \tilde{R}=\frac{R}{R_{o}}, \quad \tilde{h}=\frac{h}{h_{o}}=e(t), \quad e(t)=1-\varepsilon(t), \quad \dot{\varepsilon}=\frac{d \varepsilon}{d t}, \\
& K_{p}=\frac{r_{c}}{h_{o}}, \quad \tilde{H}_{p}=\frac{\varphi_{p} H_{p}}{h_{o}}, \quad \lambda=k\left(\frac{\mu \dot{\varepsilon} x_{o}}{h_{o}}\right)^{2}, \quad \tilde{p}=\frac{\left(p-p_{o}\right)}{\mu \dot{\varepsilon}}\left(\frac{h_{o}}{x_{o}}\right)^{2}, \quad \tilde{N}=\frac{N h_{o}^{2}}{\mu \dot{\varepsilon} x_{o}^{4}},
\end{aligned}
$$

we will obtain the formulae for the dimensionless pressure distribution and load-carrying capacity for the radial thrust bearing with a squeeze film of the Rabinowitsch type lubricant

$$
\begin{aligned}
& \tilde{p}=\frac{3}{e^{3}+\beta}\left[1-\tilde{x}^{2}-\frac{27}{10} \lambda \frac{e^{5}+\beta_{1}}{\left(e^{3}+\beta\right)^{3}}\left(1-\tilde{x}^{4}\right)\right], \\
& \tilde{N}=\frac{3 \pi}{2\left(e^{3}+\beta\right)}\left[1-\frac{18}{5} \lambda \frac{e^{5}+\beta_{1}}{\left(e^{3}+\beta\right)^{3}}\right],
\end{aligned}
$$

where

$$
\beta=\frac{3}{2} K_{p}^{2} H_{p}, \quad \beta_{l}=\frac{5}{3} K_{p}^{4} H_{p}
$$

Plots of the dimensionless pressure distribution and load carrying capacity are presented in Figs 4 and 5. 


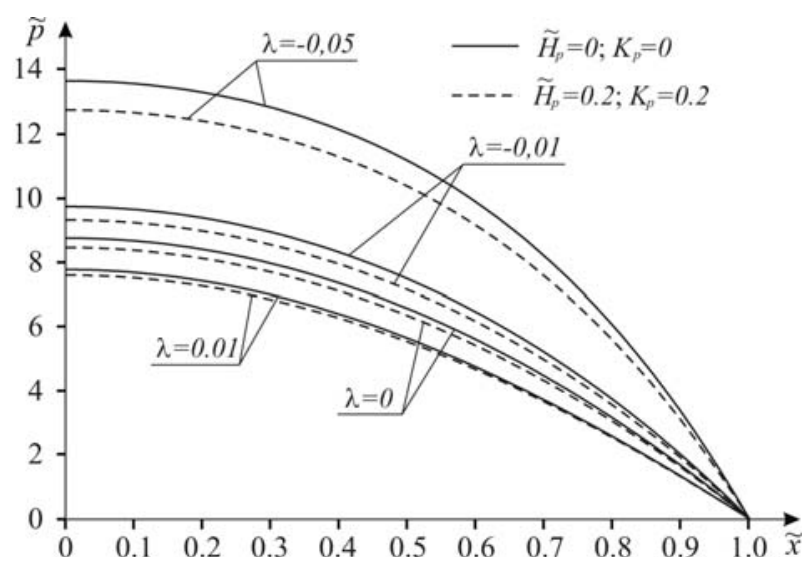

Fig.4. Dimensionless pressure distribution in the thrust bearing for $\varepsilon=0.3$.

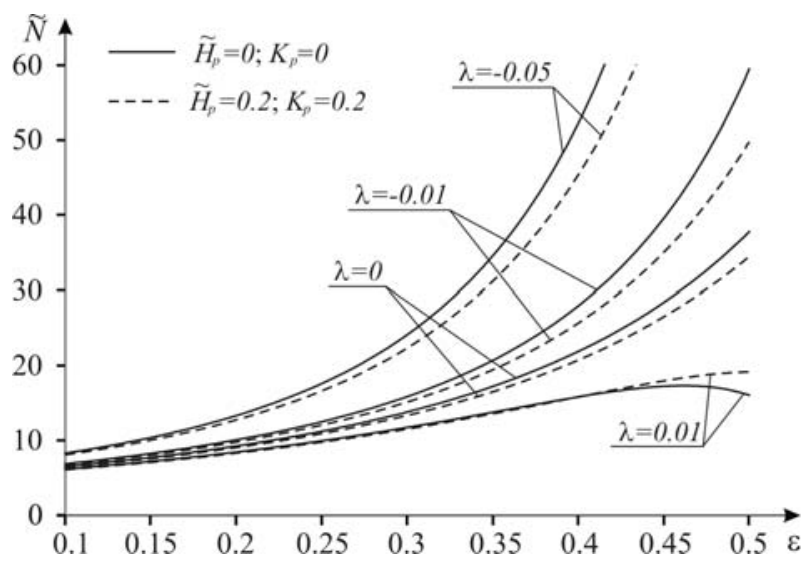

Fig.5. Dimensionless load-carrying capacity for the thrust bearing.

Figure 4 presents the dimensionless pressure $\tilde{p}$ as a function of the radial coordinate $\tilde{x}$ for a definite value of squeezing ratio $\varepsilon=0.3$ and definite values of the relative thickness of porous pad $\tilde{H}_{p}=0$ or 0.2 and definite values of the relative capillarity $K_{p}=0$ or 0.2 .

In comparison with the case of non-porous squeeze film (solid lines in Fig.4) it may be concluded that the influence of porosity decreases the film pressure with the increase of $\lambda$, for $\lambda<0$, and inconsiderably increases the film pressure with the increase of $\lambda$, for $\lambda \geq 0.01$. The influence of the pad porosity is significant for small values of $\lambda$, for $\lambda<-0.05[5-8,12-14]$

The comparison with the case of Newtonian lubricants $(\lambda=0)$ generally shows that the dilatant effects $(\lambda<0)$ increase the film pressure but the pseudo-plastic effects $(\lambda>0)$ decrease the film pressure [12-14].

Figure 5 presents the load-carrying capacity $\tilde{N}$ as a function of the squeezing ratio $\varepsilon$. This load capacity is similarly induced by dimensionless coefficient of pseudo-plasticity $\lambda$ and by parameters $K_{p}$ and $\tilde{H}_{p}$ relative to the pad porosity as the pressure distributions [1, 12-14, 22-24].

Note that all these results are partially similar to cited here earlier works concerning either the Rabinowitsch flows in bearing clearances with impermeable walls or the Newtonian flows in bearings with porous walls. 


\section{Spherical squeeze film bearing}

Let us consider now a spherical squeeze film bearing shown in Fig.6. Introducing the following parameters

$$
\begin{aligned}
& u=\tilde{h}=\frac{h}{C}=1-\varepsilon \cos \varphi, \quad u_{o}=1-\varepsilon \cos \varphi_{o}, \quad \frac{\partial \tilde{h}}{\partial t}=-\dot{\varepsilon} \cos \varphi, \quad e=1-\varepsilon, \\
& K_{p}=\frac{r_{c}}{C}, \quad \tilde{H}_{p}=\frac{\varphi_{p} H_{p}}{C}, \quad \lambda=k\left(\frac{\mu \dot{\varepsilon} R_{r}}{C}\right)^{2}, \quad \tilde{p}=\frac{\left(p-p_{o}\right) C^{2}}{\mu \dot{\varepsilon} R_{r}^{2}}, \quad \tilde{N}=\frac{N C^{2}}{\mu \dot{\varepsilon} R_{r}^{4}},
\end{aligned}
$$

we will obtain the following formulae for pressure distribution and load-carrying capacity

$$
\begin{aligned}
& \tilde{p}=\frac{6}{\varepsilon}\left[F\left(u_{o}\right)-F(u)\right], \\
& \tilde{N}=\frac{6 \pi}{\varepsilon^{3}}\left\{\left(e^{2}-2 e\right)\left[F\left(u_{o}\right)-F(e)\right]+W\left(u_{o}\right)-W(e)\right\}
\end{aligned}
$$

where

$$
F(u)=I(u)-\frac{27}{5 \varepsilon^{2}} \lambda J(u)
$$

Functions $I(u), J(u)$ and $W(u)$ are given in the Appendix B.

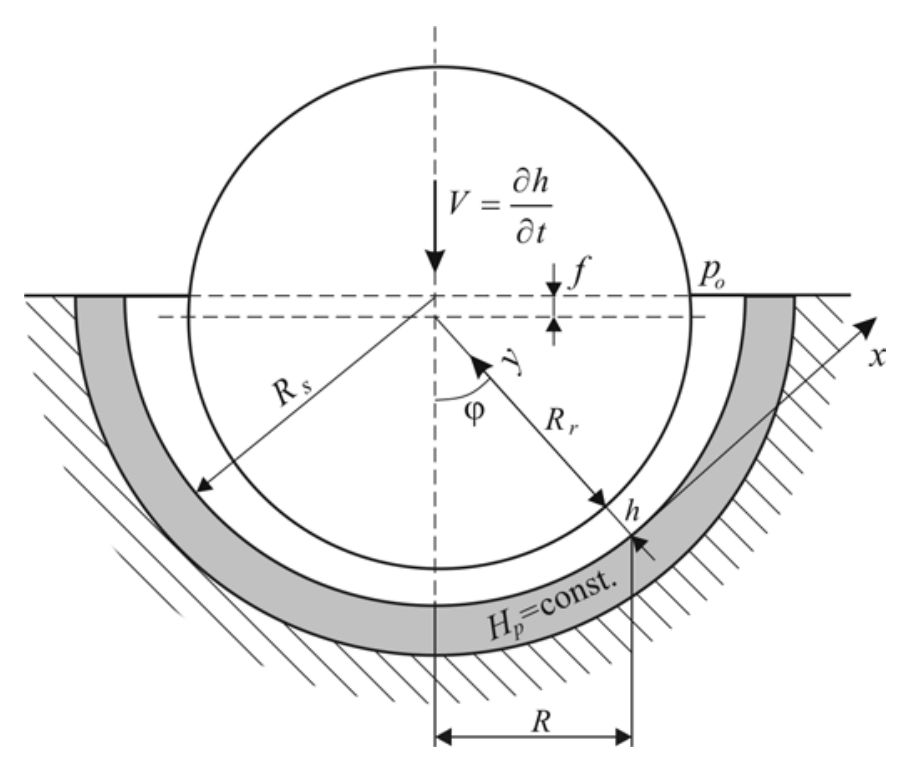

$$
\begin{aligned}
& C=R_{s}-R_{r}, \quad \varepsilon(t)=\frac{f}{C}, \\
& h=C(1-\varepsilon \cos \varphi), \\
& \varphi=\frac{x}{R_{r}}, \quad R=R_{r} \sin \varphi .
\end{aligned}
$$

Fig.6. Spherical squeeze film bearing (for $\varphi_{o}=\frac{\pi}{2}$ ). 
Figure 7 shows the dimensionless pressure $\tilde{p}$ as a function of the bearing central angle $\varphi$ for a definite value of the dimensionless bearing eccentricity $\varepsilon=0.3$ and definite values of the relative thickness of porous pad $\tilde{H}_{p}=0$ or 0.2 and definite values of the relative capillarity $K_{p}=0$ or 0.2 . It can be noted that the pressure changes run similarly to the previous bearing case but here the maximal pressure values are a bit higher and the influence of the pad porosity is a bit smaller.

Figure 8 shows the load-carrying capacity $\tilde{N}$ as a function of $\varepsilon$. This load capacity is similarly induced by the dimensionless coefficient of pseudo-plasticity $\lambda$ and by parameters $K_{p}$ and $\tilde{H}_{p}$ relative to the pad porosity. It seems that due to smaller "filling" of the pressure plots the load-carrying capacity of the spherical bearing is smaller than that of the thrust radial bearing.

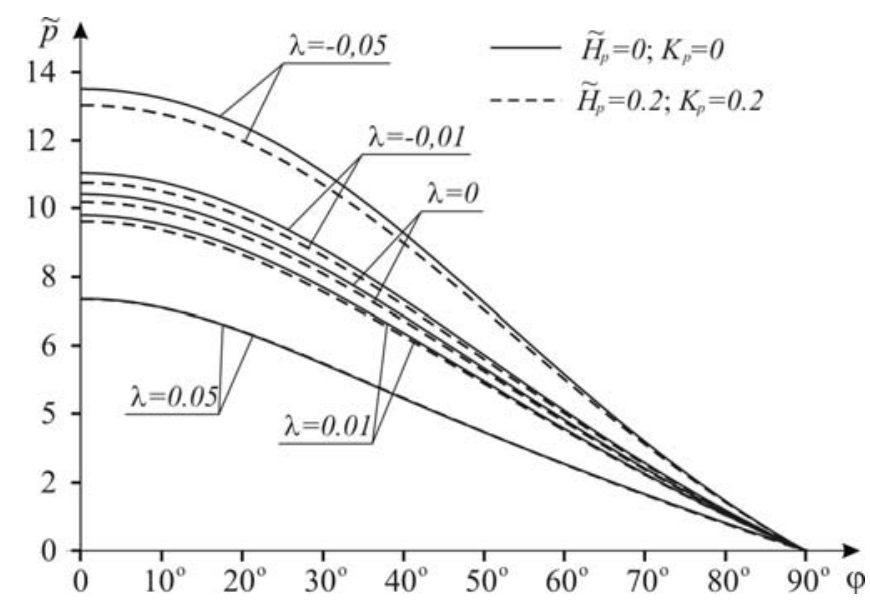

Fig.7. Dimensionless pressure distribution in the spherical bearing for $\varepsilon=0.3$.

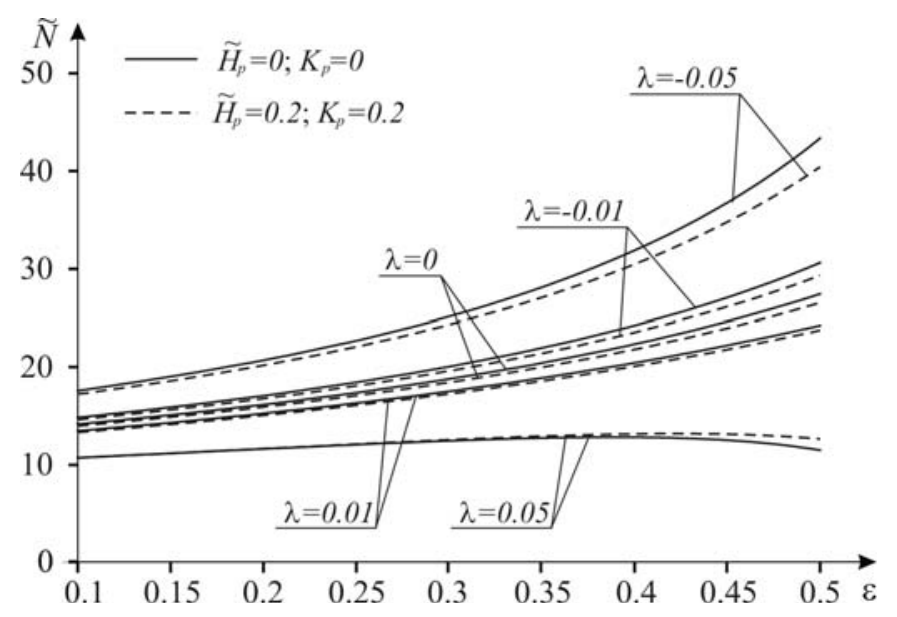

Fig.8. Dimensionless load-carrying capacity for the spherical bearing.

\section{Conclusions}

The modified Reynolds equations for a curvilinear squeeze film bearing with one porous wall lubricated by a Rabinowitsch fluid is derived. The general solution for this equation is searched in a form of the sum of two components; the first of them represents the Newtonian approximation by the second one represents the non-Newtonian correction. The detailed solution for squeeze film bearings are given for two 
cases of geometry, namely for radial thrust bearing and for spherical bearing. As a result the formulae for dimensionless pressure distributions $\tilde{p}$ and load-carrying capacity $\tilde{N}$ are obtained.

It follows from carried out calculations and their graphic presentations that the both magnitudes are dependent on the signs of rheological parameters $k$ or $\lambda$. These magnitudes are also induced by two parameters $K_{p}$ and $\tilde{H}_{p}$ relative to the bearing porous pad. The pressures and capacities increase with the decrease of the dimensionless coefficient of pseudo-plasticity $\lambda$. These magnitudes decrease with the increase of the relative thickness of porous pad $\tilde{H}_{p}$ and the relative capillarity $K_{p}$. This behaviour is typical for lubrication phenomenon of the bearings with one porous surface and it is independent on the rheological characteristic of lubricant $[1,20-24,26]$.

\section{Nomenclature}

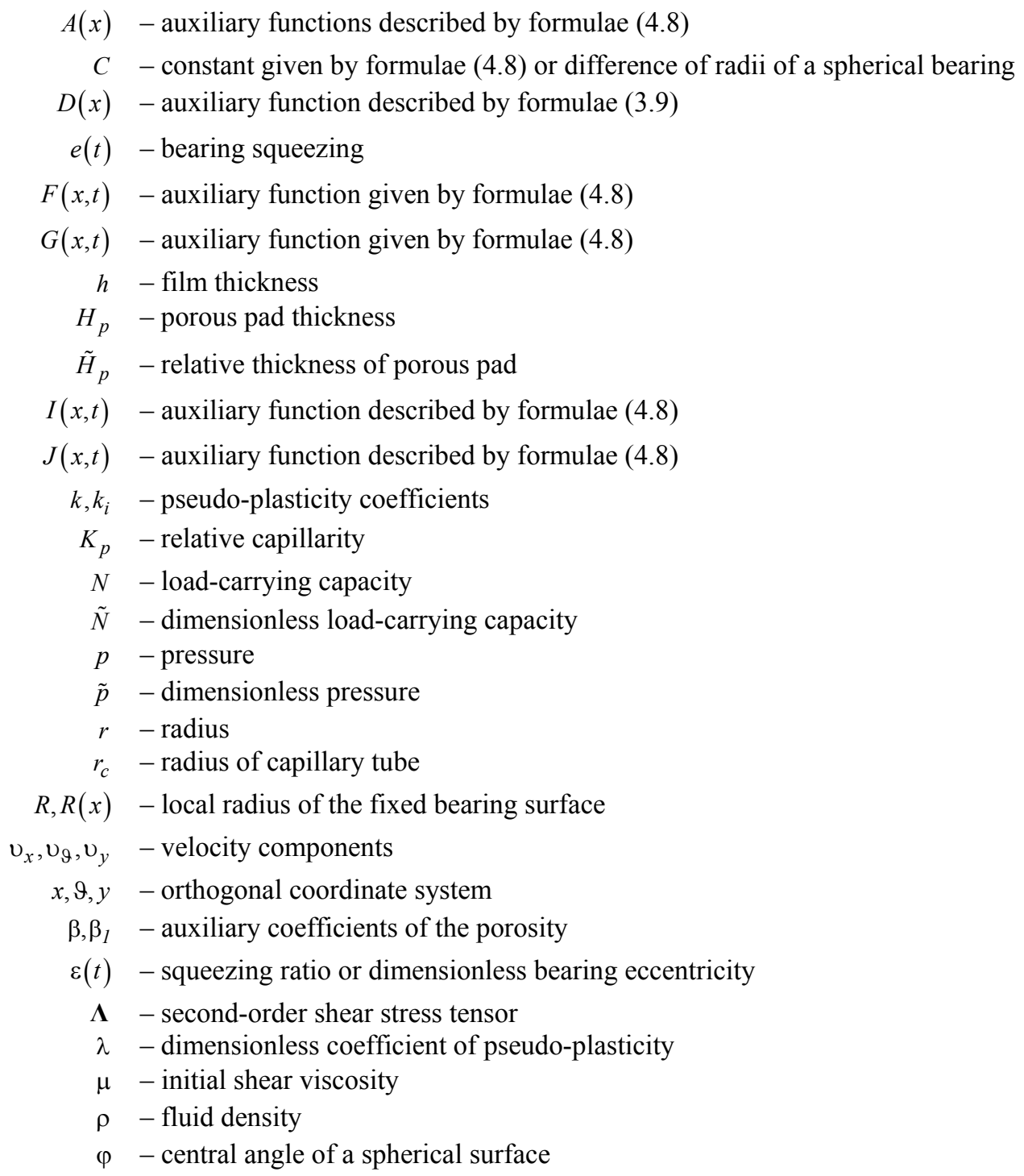

\section{References}

[1] Wu H. (1978): A review of porous squeeze films. - Wear, vol.47, pp.371-385.

[2] Kraemer E.O. and Williamson R.V. (1929): Internal friction and the structure of ,, solvated” colloids. - J. Rheology, vol.1, No.1, pp.76-92. 
[3] Rabinowitsch B. (1929): Über die Viskosität und Elastizität von Solen (On the viscosity and elasticity of sols). Zeit. Phys. Chem., A145, pp.1-26.

[4] Rotem Z. and Shinnar R. (1961): Non-Newtonian flow between parallel boundaries in linear movements. - Chem. Eng. Sci., vol.15, pp.130-143.

[5] Wada S. and Hayashi H. (1971): Hydrodynamic lubrication of journal bearings by pseudo-plastic lubricants. - (Pt 1, Theoretical studies), Bull. JSME, vol.14, No.69, pp.268-278.

[6] Wada S. and Hayashi H. (1971): Hydrodynamic lubrication of journal bearings by pseudo-plastic lubricants. - (Pt 2, Experimental studies), Bull. JSME, vol.14, No.69, pp.279-286.

[7] Swamy S.T.N., Prabhu B.S. and Rao B.V.A. (1975): Stiffness and damping characteristics of finite width journal bearing with a non-Newtonian film and their application to instability prediction. - Wear, vol.32, pp.379-390.

[8] Rajalingham C., Rao B.V.A. and Prabu S. (1978): The effect of a non-Newtonian lubricant on piston ring lubrication. - Wear, vol.50, pp.47-57.

[9] Sharma S.C., Jain S.C. and Sah P.L. (2000): Effect of non-Newtonian behaviour of lubricant and bearing flexibility on the performance of slot-entry journal bearing. - Tribology Int., vol.33, pp.507-517.

[10] Singh U.P., Gupta R.S. and Kapur V.K. (2011): On the steady performance of hydrostatic thrust bearing: Rabinowitsch fluid model. - Tribology Transactions, vol.54, pp.723-729.

[11] Hashimoto H. and Wada S. (1986): The effects of fluid inertia forces in parallel circular squeeze film bearing lubricated with pseudoplastic fluids. - J. Tribology, vol.108, pp.282-287.

[12] Lin J.-R. (2012): Non-Newtonian squeeze film characteristics between annular disks: Rabinowitsch fluid model. Tribology Int., vol.52, pp.190-194.

[13] Lin J.-R., Chu L.-M., Hung C.-R., Lu R.-F. and Lin M.-C. (2013): Effects of non-Newtonian rheology on curved circular squeeze film: Rabinowitsch fluid model. - Z. Naturforsch., vol.68a, pp.291-299.

[14] Walicka A., Walicki E. and Ratajczak M. (1999): Pressure distribution in a curvilinear thrust bearing with pseudo-plastic lubricant. - Appl. Mech. Enging., vol.4 (sp. Issue), pp.81-88.

[15] Walicka A., Walicki E. and Ratajczak M. (2000): Rotational inertia effects in a pseudo-plastic fluid flow between non-coaxial surfaces of revolution. - Proc. 4th Minsk Int. Heat Mass Transfer Forum (May 22-27, 2000 Minsk Belarus), pp.19-29.

[16] Ratajczak M., Walicka A. and Walicki E. (2006): Inertia effects in the curvilinear thrust bearing lubricated by a pseudo-plastic fluid of Rotem-Shinnar. - Problems of Machines Expoitation, vol.44, pp.159-170.

[17] Walicka A. and Walicki E. (2010): Performance of the curvilinear thrust bearing lubricated by a pseudo-plastic fluid of Rotem-Shinnar. - Int. J. Appl. Mech. Enging, vol.15, pp.895-907.

[18] Morgan V.T. and Cameron A. (1957): Mechanisms of lubrication in porous metal bearings. - Proc. Conf. on Lubrication and Wear, Inst. Mech. Eng., London, pp.151-157.

[19] Gupta R.S. and Kapur V.K. (1979): Centrifugal effects in hydrostatic porous thrust bearings. - J. Lubric. Technology, vol.101, pp.381-392.

[20] Prakash J. and Vij S.K. (1974): Analysis of narrow porous journal bearing using Beaver-Joseph criterion of velocity slip. - J. Appl. Mech., vol.41, pp.348-354.

[21] Uma S. (1974): The analysis of double layered porous slider bearing. - Wear, vol.42, pp.205-215.

[22] Walicka A. (2012): Porous curvilinear squeeze film bearing with rough surfaces lubricated by a power-law fluid.Journal of Porous Media, vol.15, No.1, pp.29-49.

[23] Walicka A. (2013): Pressure distribution in a squeeze film of a Shulman fluid between porous surfaces of revolution. - Int. J. Engng Sci., vol.69, pp.33-48.

[24] Walicka A. and Jurczak P. (2013): Pressure distribution in a porous squeeze film bearing lubricated by a Vočadlo fluid. - Appl. Mathematical Modelling, vol.37, No.22, pp.9295-9307. 
[25] Walicka A. (2002): Rotational Flows of Rheologically Complex Fluids in Thin Channels (in Russian). - Zielona Gora: University Press.

[26] Walicki E. (2005): Rheodynamics of Slide Bearings Lubrication (in Polish). - Zielona Gora: University Press.

[27] Wada S., Nishiyama N. and Nishida S. (1985): Modified Darcy's law for non-Newtonian fluid. - Bulletin JSME, vol.28, pp.3031-3037

[28] Rajalingham C., Rao B.V.A. and Prabu B.S. (1979): Steady state performance of a hydrodynamic journal bearing with a pseudo-plastic lubricant. - J. Lubric. Technol., vol.101, pp.497-502.

Received: November 12, 2016

Revised: January 2, 2017

\section{Appendix A}

The flow of a Rabinowitsch fluid in a porous layer satisfies the modified Darcy's law (3.1). This flow should also satisfies the following equation of continuity

$$
\frac{1}{R} \frac{\partial\left(R \overline{\mathrm{v}}_{x}\right)}{\partial x}+\frac{R \overline{\mathrm{v}}_{y}}{\partial y}=0 .
$$

Substituting Eqs (3.1) into Eq. (A.1) one obtains an analogue of the Laplace equation

$$
\frac{1}{R} \frac{\partial}{\partial x} R\left\{\left(-\frac{\partial \bar{p}}{\partial x}\right)+\frac{k r_{c}^{2}}{6}\left(-\frac{\partial \bar{p}}{\partial x}\right)^{3}\right\}+\frac{\partial}{\partial y}\left\{\left(-\frac{\partial \bar{p}}{\partial y}\right)+\frac{k r_{c}^{2}}{6}\left(-\frac{\partial \bar{p}}{\partial y}\right)^{3}\right\}=0 .
$$

Integrating Eq. (A.2) with respect to $y$ over the porous layer of thickness $H_{p}$ one obtains

$$
\left.\left\{\left(-\frac{\partial \bar{p}}{\partial y}\right)+\frac{k r_{c}^{2}}{6}\left(-\frac{\partial \bar{p}}{\partial y}\right)^{3}\right\}\right|_{y=0}=-\frac{1}{R} \frac{\partial}{\partial x} R \int_{-H_{p}}^{0}\left\{\left(-\frac{\partial \bar{p}}{\partial x}\right)+\frac{k r_{c}^{2}}{6}\left(-\frac{\partial \bar{p}}{\partial x}\right)^{3}\right\} d y
$$

since

$$
\left.\left\{\left(-\frac{\partial \bar{p}}{\partial y}\right)+\frac{k r_{c}^{2}}{6}\left(-\frac{\partial p}{\partial y}\right)^{3}\right\}\right|_{y=-H_{p}}=0
$$

as the porous facing is press-fitted in a solid housing as shown in Fig.2.

Substituting

$$
\frac{1}{R} \frac{\partial}{\partial x} R\left\{\left(-\frac{\partial \bar{p}}{\partial x}\right)+\frac{k r_{c}^{2}}{6}\left(-\frac{\partial \bar{p}}{\partial x}\right)^{3}\right\}=f(x, y)
$$

we have

$$
\int f(x, y) d y=F(x, y)
$$


taking into account (A.3) one may write

$$
\left.\left\{\left(-\frac{\partial \bar{p}}{\partial y}\right)+\frac{k r_{c}^{2}}{6}\left(-\frac{\partial \bar{p}}{\partial y}\right)^{3}\right\}\right|_{y=0}=-\left\{F(x, 0)-F\left(x,-H_{p}\right)\right\} .
$$

But

$$
\lim _{H_{p} \rightarrow 0} \frac{F(x, 0)-F\left(x,-H_{p}\right)}{H_{p}}=\left.\left\{\left(-\frac{\partial \bar{p}}{\partial y}\right)+\frac{k r_{c}^{2}}{6}\left(-\frac{\partial \bar{p}}{\partial y}\right)^{3}\right\}\right|_{y=0}=f(x, 0) .
$$

Therefore

$$
\begin{aligned}
& \left.\lim _{H_{p} \rightarrow 0}\left\{\left(-\frac{\partial \bar{p}}{\partial y}\right)+\frac{k r_{c}^{2}}{6}\left(-\frac{\partial \bar{p}}{\partial y}\right)^{3}\right\}\right|_{y=0}=-H_{p} f(x, 0)= \\
& =-\left.\frac{H_{p}}{R} \frac{\partial}{\partial x} R\left\{\left(-\frac{\partial \bar{p}}{\partial x}\right)+\frac{k r_{c}^{2}}{6}\left(-\frac{\partial \bar{p}}{\partial x}\right)^{3}\right\}\right|_{y=0}=-\frac{H_{p}}{R} \frac{\partial}{\partial x} R\left\{\left(-\frac{\partial \bar{p}}{\partial x}\right)+\frac{k r_{c}^{2}}{6}\left(-\frac{\partial \bar{p}}{\partial x}\right)^{3}\right\}
\end{aligned}
$$

since the pressure is continuous at the porous wall-fluid interface and the pressure in the squeeze film is independent of $y$. Taking into account (A.9) into (A.3) one obtains Eq.(3.4).

\section{Appendix B}

$$
\begin{aligned}
& I(u)=-\frac{1}{6 \beta^{2 / 3}}\left(2 I_{01}-2 \ln N+\ln M\right) ; \quad \beta=\frac{3}{2} K_{p}{ }^{2} \tilde{H}_{p}, \quad \beta_{1}=\frac{5}{3} K_{p}{ }^{4} \tilde{H}_{p} \\
& J(u)=-\left(1-\varepsilon^{2}\right) \beta_{1} J_{1}+2 \beta_{1} J_{2}-\beta_{1} J_{3}-\left(1-\varepsilon^{2}\right) J_{4}+2 J_{5}-J_{6} \\
& J_{1}=\frac{1}{243 \beta^{11 / 3}}\left[\frac{27 u \beta^{8 / 3}}{\left(u^{3}+\beta\right)^{3}}+\frac{36 u \beta^{5 / 3}}{\left(u^{3}+\beta\right)^{2}}+\frac{60 u \beta^{2 / 3}}{u^{3}+\beta}-40 I_{01}-40 \ln N-20 \ln M\right] \\
& J_{2}=\frac{1}{486 \beta^{10 / 3}}\left[\frac{54 u^{2} \beta^{7 / 3}}{\left(u^{3}+\beta\right)^{3}}+\frac{63 u^{2} \beta^{4 / 3}}{\left(u^{3}+\beta\right)^{2}}+\frac{84 u^{2} \beta^{1 / 3}}{u^{3}+\beta}-28 I_{01}-28 \ln N+14 \ln M\right] \\
& J_{3}=-\frac{1}{9\left(u^{3}+\beta\right)^{3}}, \quad J_{4}=-\frac{3 u^{3}+\beta}{18\left(u^{3}+\beta\right)^{3}}
\end{aligned}
$$




$$
\begin{aligned}
& J_{5}=\frac{1}{243}\left[\frac{27 u \beta}{\left(u^{3}+\beta\right)^{3}}+\frac{45 u}{\left(u^{3}+\beta\right)^{2}}+\frac{6 u}{\beta\left(u^{3}+\beta\right)}+\frac{1}{\beta^{5 / 3}}\left(-4 I_{01}+4 \ln N-2 \ln M\right)\right], \\
& J_{6}=\frac{1}{486}\left[\frac{54 u^{2} \beta}{\left(u^{3}+\beta\right)^{3}}-\frac{99 u^{2}}{\left(u^{3}+\beta\right)^{2}}+\frac{30 u^{2}}{\beta\left(u^{3}+\beta\right)}+\frac{1}{\beta^{4 / 3}}\left(-10 I_{01}+10 \ln N-5 \ln M\right)\right], \\
& I_{01}=\sqrt{3} \arctan \frac{\beta^{1 / 3}-2 u}{\sqrt{3} \beta^{1 / 3}}, \quad N=u^{3}+\beta, \quad M=u^{2}-\beta^{I / 3} u+\beta^{2 / 3}, \\
& W(u)=2 I_{1}(u)-\frac{1}{3} \ln N-\frac{27 \lambda}{5 \varepsilon^{2}}\left[-2\left(1-\varepsilon^{2}\right) \beta_{1} W_{1}+\left(5-\varepsilon^{2}\right) \beta_{1} W_{2}-4 \beta_{1} W_{3}+\right. \\
& \left.+\beta_{1} W_{4}-2\left(1-\varepsilon^{2}\right) W_{5}+\left(5-\varepsilon^{2}\right) W_{6}-4 W_{7}+W_{8}\right], \\
& I_{l}(u)=-\frac{1}{6 \beta^{1 / 3}}\left(2 I_{01}-2 \ln N+\ln M\right), \\
& W_{1}=\frac{1}{486 \beta^{10 / 3}}\left(\frac{54 u^{2} \beta^{7 / 3}}{N}+\frac{63 u^{2} \beta^{4 / 3}}{N}+\frac{84 u^{2} \beta^{1 / 3}}{N}-28 I_{01}-28 \ln N+14 \ln M\right), \\
& W_{2}=-\frac{1}{9 N}, \\
& W_{3}=\frac{1}{486}\left[-\frac{54 u}{N^{3}}+\frac{9 u}{\beta N^{2}}+\frac{15 u}{\beta^{2} N}+\frac{1}{\beta^{8 / 3}}\left(-10 I_{01}+10 \ln N-5 \ln M\right)\right] \text {, } \\
& W_{4}=\frac{1}{243}\left[-\frac{27 u^{2}}{N^{3}}+\frac{9 u^{2}}{\beta N^{2}}+\frac{12 u^{2}}{\beta^{2} N}+\frac{1}{\beta^{7 / 3}}\left(-4 I_{01}-4 \ln N+2 \ln M\right)\right] \text {, } \\
& W_{5}=\frac{1}{243}\left[\frac{27 u \beta}{N^{3}}-\frac{45 u}{N^{2}}+\frac{6 u}{\beta N}+\frac{1}{\beta^{5 / 3}}\left(-4 I_{01}+4 \ln N-2 \ln M\right)\right] \text {, } \\
& W_{6}=\frac{1}{486}\left[\frac{54 u^{2} \beta}{N^{3}}-\frac{99 u^{2}}{N^{2}}+\frac{30 u^{2}}{\beta N}+\frac{1}{\beta^{4 / 3}}\left(-10 I_{01}-10 \ln N+5 \ln M\right)\right] \text {, } \\
& W_{7}=-\frac{3 u^{6}+3 u^{3} \beta+\beta^{2}}{9 N^{3}} \\
& W_{8}=\frac{1}{486}\left[-\frac{54 u \beta^{2}}{N^{3}}+\frac{171 u \beta}{N^{2}}-\frac{201 u}{N}+\frac{1}{\beta^{2 / 3}}\left(-28 I_{01}+28 \ln N-14 \ln M\right)\right] .
\end{aligned}
$$

